\title{
Téoros
}

Revue de recherche en tourisme

\section{Les trois espaces de la carte du touriste selon Jean-Didier Urbain : contribution à l'étude touristique des relations centres et périphéries}

\author{
Marc Laplante
}

Volume 13, numéro 1, mars 1994

Bas-Saint-Laurent, Gaspésie, Iles-de-la-Madeleine : fascinantes périphéries

URI : https://id.erudit.org/iderudit/1077776ar

DOI : https://doi.org/10.7202/1077776ar

Aller au sommaire du numéro

Éditeur(s)

Université du Québec à Montréal

ISSN

0712-8657 (imprimé)

1923-2705 (numérique)

Découvrir la revue

Citer cet article

Laplante, M. (1994). Les trois espaces de la carte du touriste selon Jean-Didier Urbain : contribution à l'étude touristique des relations centres et périphéries.

Téoros, 13(1), 40-45. https://doi.org/10.7202/1077776ar d'utilisation que vous pouvez consulter en ligne. 


\section{Les trois espaces de la carte du touriste selon Jean-Didier Urbain: contribution à l'étude touristique des relations centres et périphéries Marc Laplante *}

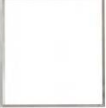

Parmi les interrogations à l'origine du présent colloque, nous trouvons la question suivante: comment vendre touristiquement une périphérie, un bout du monde? On a tenté d'y répondre de diverses manières en réexaminant les habitudes de pensée de la recherche en marketing ou encore, en traduisant de façon opératoire des modèles de l'approche géographique du tourisme.

Pour ma part, j'ai cherché à apporter une contribution complémentaire à ces approches en exploitant un champ vaste et peu utilisé encore: celui des relations entre espace et imaginaire. La sociologie de la culture qui meguide pour cet essai - a déjà exploré ce champ, notamment par des travaux en communication. Ceux-ci ont commencé à se distinguer en recherche sous le thème de la sémiologie spatiale.

Je ne peux pas, dans le cadre de cet exposé, rappeler les acquis dans ce domaine ni poser les éléments théoriques qui les fondent. Mais, puisque nous nous occupons du tourisme, je dispose d'un ouvrage récent produit par un chercheur qui maîtrise bien cedomaine des sciences de la communication et qui s'en est servi pour analyser longuement le touriste et le tourisme: il s'agit du livre de Jean-Didier Urbain publié chez Plon, en France, en 1991 et intitulé: L'idiot du voyage. Histoires de touristes.

Comme l'idiot du village qui n'est jamais aussi fou qu'on aime à le dire et qui sert socialement de faire-valoir à ceux qui se veulent intelligents ou rusés, l'idiot du voyage est le touriste au sens historique du terme: celui qui voyage pour le plaisir de voyager, qui fait ce qu'on appelle aujourd'hui du nomadisme de loisir ou des voyages d'agrément.
Monsieur Marc Laplante est professeur au département d'études urbaines et touristiques de rUniversité du Québec à Montréal et directeur des publications Téoros sur la recherche en tourisme.

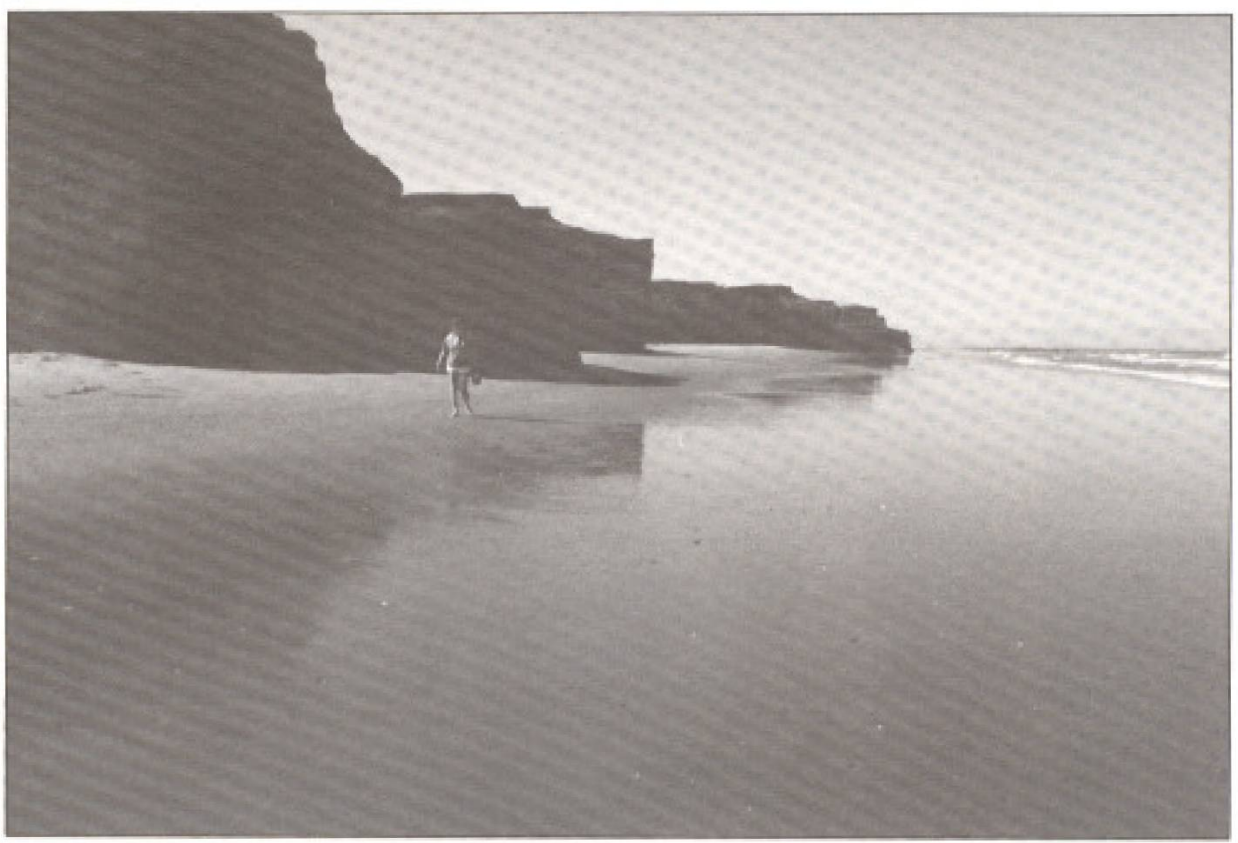

Plus on avance dans la traversée de l'Est du Québec, plus on va vers un bout du monde...

Photo : Marc Laplante.

L'idiotduvoyagen'estpas aussi idiotqu'on aimerait - et qu'on aime toujours - à le dire. Mais il a tellement entendu les autres voyageurs le traiter d'idiot qu'il a peine maintenant à se défaire lui-même de cette étiquette négative qu'on lui a collé sur la peau: beaucoup de touristes d'aujourd'hui essaient encore et toujours de ne pas être touristes, de ne pas agir en touriste, etc. J.D. Urbain consacre une bonne partie de son livre - la première et la plus longue - à retracer à travers plus de trois siècles de litterratures sur les voyages et les touristes les fondements de cette dichotomie opposant le voyageur et le touriste ${ }^{(1)}$. Evidemment, le grand intérêt du livre de J.-D. Urbain tient précisément dans sa belle démonstration que le touriste n'est pas aussi idiot qu'on pense (et que les idiots sont peut-êtreplutốt du côté de ceux quiles pensent!).

Cette démonstration est faite, en bonne partie, dans la seconde section de l'ouvrage intitulée: «La carte du touriste. Histoire d'espaces». Mes collègues de géographie se réjouissent déjà d'entendre que la réhabilitation du touriste passe par la voie obligée d'une histoire d'espaces. Ils n'ont pas tort mais je souhaite qu'ils entreprennent leur propre critique de cette oeuvre au-delà de ce que je vais essayer d'en tirer ici pour les fins de ce colloque.

Je propose de tirer d'abord des premières pages de cette seconde partie de l'ouvrage de J.-D. Urbain certaines propositions qui rendront plus clairessa démarche. Ensuite, j'essaieraideles commenter pour introduire quelques questions utiles à notre réflexion communesur les façons souhaitables d'offrir la péninsule gaspésienne aux touristes.

\section{Énoncés d'introduction au système de territoires symboliques}

Nous pouvons, je crois, acquérir une bonne compréhension des visées et de la démarche del'auteur avec les cinq énoncés suivants tirés textuellement de son livre:

a) «Les expériences touristiques sont des expériences transversales qu'on ne peut confinerdans des espacesspécifiques.» (p. 110) 
b) «Le tourisme (...) présuppose un imaginaire de l'espace. Cet imaginaire donne sens à la airculation touristique et differencie ses tribus, $\%$ (p. 110)

c) *Cen'estpasun plan derépartitionde la population touristique sur la planète qu'on tracera mais un système de territoires symboliques quel'on construira, ion de lassociation de modeles d'epacesàdervaleurs esentielless (p. 111)

d) *Ce système, c'est la carte du touriste, plus proche de la carte du tendre de mademoiselle de Scudéry que de la géographie $\rightarrow$ (p. 111)

e) sLe rapport entre espaces et imaginaire est ici privilégié, définissant un ensemble de lieut invertis par les mythologies vacancières, sources d'innombrables nomadismes... Comme le dit l'anthropologue E.T.Hall, le langage de la culture est aussi clair que celui des rêves analysés par Freud, à cette différence près que les grands songes culturels ne s'expriment pas tant à travers ce que les gens disent qu'à travers ce qu'ils font. Ceci est vrai dut tourime dont l'usage des expaces est aussi un langagess (p. 111 )

Jean-Didier Urbain est donc parti à la recherche du système de territoires symboliques formant la carte du touriste, carte des grands songes culturels qui se sont exprimésà travers les âges par divers usages des espaces. Il a reconstitué alors le code qui peut nous aider à comprendre ce langage des usages de l'espace élaboré par de nombreux nomadismes.

Arrêtons-nous d'abord aux énoncés présentés ci-haut; en les commentant, j'introduirai certainesquestions utiles - jecrois - à notre réflexion commune sur les façons souhaitables d'offrir la péninsule gaspésienne aux touristes.

\section{Les expériences touristiques sont des expériences transversales}

On ne peut pas, selon J.-D. Urbain, confiner les expériences touristiques dans des espaces spécifiques. Pratiquement, ceci veut dire qu'on ne peut plus parler de tourisme de montagne, rural, balnéaire, urbain, etc. Transversales, ces expériences lesont parce qu'elles exigent la circulation et le passage au travers des espaces différents, différents symboliquement, mythologiquement, autant - sinon plus - que matériellement. Il faut préciser ici quel'auteur ne reconnaît pas comme touriste le vacancier sédentaire hors de son domicile, c'est-à-dire le villégiateur coutumier qui se transplante dansun lieu devacances sans en bouger. Le touriste est obligatoirement celui qui circule, le nomade. Je partage entièrement cette définition après recherche sur l'origine du concept de touriste, de l'individu ainsi désigné et des expériences qu'il vit.

J.-D. Urbain met ainsi en doute l'intérêt et l'utilité de nombreuses typologies et catégorisations qui sont monnaie courante dans la gestion touristique. Personnellement, j'essaie depuis quelques années de comprendre ce qu'on désigne comme tourisme urbain; le tourisme rural n'est pas plus évident; ces catégories désignent des sous-ensembles de produits et services localisés en tel ou tel lieu sans considérer le sens de ces lieux, le agéniedu lieus, comme disent maintenant les spécialistes du patrimoine. La carte du touriste nous libère d'abord de ces typologies trop élémentaires et évidentes qui sont souvent trompeuses; elle nous introduit aussi à autres choses comme nous allons le voir.

\section{Le tourisme présuppose l'imaginaire de l'espace}

Cet imaginaire de l'espace s'est construit avant la naissance du tourisme moderne, sur de longues périodes historiques. Ramifié, modifié, cet imaginaire est toujours à l'oeuvre aujourd'hui et agit souvent directement sur les idées, les choix et les actions des touristes.

J.-D. Urbain retient, pour sa recherche, la période de la Renaissance (XVI ${ }^{*}$ siècle) à nos jours en Occident mais il réfere parfois à une littérature du voyage aussi lointaine que le livre de Marco Polo parut entre 1298 et 1300. Les diverses catégories de touristes vont se constituer historiquement en puisant dans cet imaginaire de base, sorte d'univers symbolique que chaque catégorie et chaque époque vont accommoder à leurs besoins et à leurs désirs. La circulation touristique, en segreffant ainsi, dès ses débuts, sur un imaginaire de l'espace richement étoffé par plusieurs siècles de récits de voyageurs, s'inscrit d'emblée comme une expérience culturelle.
Pratiquement, les responsables de l'offre touristique devront revoir leurs démarches et donner la priorité à l'univers symbolique qui enveloppe les territoires à offrir à la curiosité du touriste. Ici, dans la péninsule gaspésienne, les récits des voyageurs ne manquent pas depuis que Jacques Cartier a plantésa croixà Gaspé: ces textes devraient servir d'abord à reconstituer l'imaginaire de ces espaces pour ensuite repérer les catégories de touristes qu'il pourra séduire. Je préciserai ceci dans un moment.

\section{Un système de territoires symboliques}

Ce sujet forme la partie la plus importante du présent texte puisque son développement suscitera, en conclusion, plusieurs idées nouvelles pour réfléchir sur les relations entre le centre et la périphérie en développement touristique.

Je ne pourrai done pas m'attarder aux ếnoncés d) et e) formulés précédemment. Ảleur propos, jevoudraissimplement qu'on note à quel point ils posent des défis à la recherche en tourisme: étude des mythologies vacancières, des grands songes culturels et de leurs modes d'expression dans divers usages des espaces, etc. Pouvonsnoussimplement imaginer aujourd'hui que des subventions de recherche deviendront disponibles un jour pour aborder de tels sujets? Et pourtant, comme j'essaierai de l'illustrer ici, ces recherches pourraient être très utiles...

Il me faut résumer très schếmatiquement les thèses de J.-D. Urbain sur les systèmes de territoires symboliques pour prendre le temps, enfin, d'en souligner les implications.

\section{Des systèmes présents dans toutes les civilisations}

À titre d'exemple, l'auteur rappelle l'existence d'un tel système dans la culture grecque, décrit notamment par Hérodote. Pour les Grecs d'il y a plus de 2300 ans, partir, voyager, s'éloigner s'inscrit dans une symbolique des espaces qui voit moins le voyageur traverser des pays que parcourir une échelle qui va de l'humain au non-humain en passant par les Barbares, les nongrecs et les monstres humanoïdes des confins. Plus on s'éloigne du centre, de l'axe méditerranćen, phus on s'éloigne del l'bumain. 
Faut-il mentionner qu'un tel système de territoires symboliques a perduré presque jusqu'à nous! Un jour, le samvage est devenu, avec Jean-Jacques Rousseau, le bon sauvage mais que se cache-t-il encore aujourd'hui sous le concept de peuples primitifs, par exemple, qu'on offre toujours comme attraction touristique?

\section{Un trio d'espaces dans la civilisation occidentale depuis 1300}

En Occident, depuis Marco Polo, le système de territoires symboliques a retenu trois espaces: la ville, la campagne, le désert. *Cette composition atteste un code de perception qui assigne désormais un valeur symbolique à chaque territoire. La ville, lieu clos, protectrice, est un espace de sociabilité maximale: une concentration d'hommes; la campagne, espace ouvert, est un lieu hétérogène de faible densité: hommes et animaux y vivent ensemble; le désert, ascétique, espace extra-social, est un lieu de solitude toumé vers le divin.»(2) J.-D. Urbain s'applique alors à suivre les ramifications et les modifications de ce codedeperception installé dansl'imaginaire dela culture occidentale dès le XVII' siècle.

La ville: elle fut la première curiosité touristique. La ville était alors rassurante parce que le touriste n'avait pas en tête une arte et ne connaissait pas l'espace dans lequel il circulait. En ville, l'espace était organisé et le touriste recherchait un point élevé pour avoir un panorama de cet espace; là, il pouvait lire la ville comme lieu d'ordre; au siècle des Lumières (XVIII ${ }^{\circ}$, on aime ce qui estintelligible, ordonné. Parallèlement, hors de la ville, on trouve le danger, le désordre: la nature reste hostile, les routes sont dangereuses, etc.

Au cours du XIX' siècle, la ville perd de son importance relative mais reste privilégiée: des pays se fontconnaître par leurs grandes villes. Mais, en même temps, la campagne devientmoinsdangereuse, la naturedevient valorisée(découvertede nouveauxespaces: montagne, désert, etc.) et surtout, les villes nouvelles inquiètent tout en fascinant (villesaméricaines surtout qui poussentcomme des champignons ou des cancers, deviennent gigantesques, laissent apparaître certains désordres).

A la fin du $\mathrm{XIX}^{\mathrm{c}}$ siècle, la ville devient inquiétante eton commenceà la quitter. Elle devient émettrice de touristes.

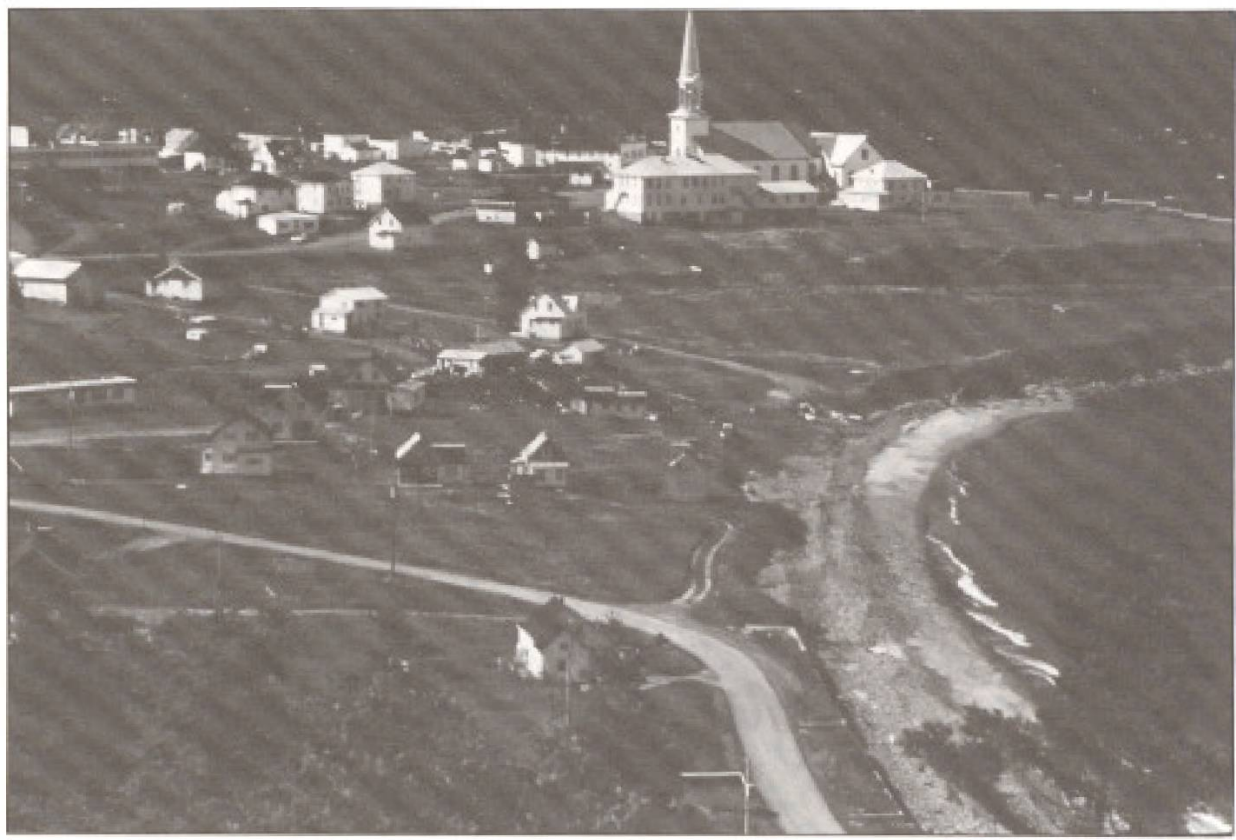

Les lieux-limites sont des attraits irrésistibles pour les touristes. Ici, au Mont St-Pierre, la campagne ct la mer se rencontrent. Photo: Pierre Saint-Jacques.

Aujourd'hui, certaines villes sont transparentes à leurs valeurs: Washington: son mémorial; Las Vegas: le jeu; Jérusalem: la religion; Bangkok: le sexe, etc. Mais la plupart des villes sont équizoques, polyvalentes, incertaines; elles attirent et repoussent à la fois et certaines sont des antidestinations ${ }^{(3)}$.

En fait, généralement, de nos jours, une ville en cache souvent une autre : la ville visible offre la sérénité de ses monuments et la ville obscure, la panique des rues; la première fait voir ses luxes, la seconde, sa luxure; aux fastes silencieux du patrimoine s'oppose la misère bruyante des quartiers. La ville d'aujourd'hui est une mosaique de territoires imbriqués les uns dans les autres et difficilement déchiffrable: elle est toujours un condensé de civilisation et aussi une jungle dangereuse $\mathrm{e}^{(+)}$.

Et la ville attire alors deux sortes de touristes: les prudents, amateurs de cimes, de visible et les téméraires, explorateurs de la jungle, de l'obscur; nous les retrouverons bientôtt.

La campagne. Au XVI' siècle, la campagne est associée à: distance, chemin, danger, fatigue. Puis, au fil du temps, cette campagne s'esquisse comme un territoire entre ville et désert, entre culture et nature, entre bourg et forêt; elle devient un tiers espace, comme un territoire autonome.
Il faut comprendre que la campagne est une invention et non une donnée qui va de soi. L. Marin a défini la campagne: «la défiguration nécessaire de la nature par l'homme social $\aleph^{(5)}$. A la campagne, lieu de transition, la nature et la culture, loin de s'annuler, setransformentl'une dansl'autre. Si la campagne apparaît finalement comme tiers espace, c'est qu'elle est née symboliquement comme territoire à mesure que les deux pôles: ville et désert s'éloignaient l'un de l'autre.

Lé désert: il commence là où la campagne cesse. Forêt et mer ont aussi fait office de désert dans l'histoire des territoires symboliques. Le désert est avant tout l'espace du divin; il est une mystique, un lieu d'initiation, d'ascèse, de croyances, d'extasé: la mortification y est récompensée, la révélation se manifeste. C'est l'espace du voyageur illuminé.

\section{Trois espaces, un système}

Chacun, en lisant les lignes qui précèdent, retrouvera dans son propre imaginaire et dans ses songes culturels des associations avec ces territoires symboliques de la ville, de la campagne ou du désert. Il nous importe maintenant de considérer ces trois univers comme un système. J.-D. Urbain, qui a écrit des pages passionnantes sur chacun d'eux, associe toujoursl'expérience 
touristique vécue réellement par divers touristes à ce système d'espaces.

Précisons d'abord, avec l'auteur, qu'aucun de ces espaces est univoque: tous sont ambivalents. Univers symboliques, ils ont évolué historiquement comme on l'a vu, par exemple, avec les changements dans le code de perceptions de la ville du XVI siècle à nos jours. De plus, les territoires symboliques ne coincident pas avec les espaces géographiques réels: par exemple, s'il y a une Gaspésie désent (mer, forêts vierges, etc.), il importe peu de savoir si elle commence après Rimouski ou après SainteAnne-des-Monts, Il faudra la chercher d'abord dans desrécits de voyageurs depuis que cette péninsule est visitéc; de même pour la Gaspésie-campagne ou même la Gaspésie-ville. Par la suite, on pourra l'offrir comme territoire symbolique en sachant que cet imaginaire rejoindra différemment telleou telle tribu touristique.

J.-D. Urbain emploie l'expression tribu touristique pour échapper aux catégories habituelles de clientèles. «Une tribu est, à l'intérieur d'une culture, unemicro-société autonome qui se singularise par un certain nombre de signes spécifiques [...] $]$ (p. 107). La tribu touristique est une micro-société éphémère du temps des vacances. En introduction de la section du livre portant sur la carte du touriste, l'auteur parle de *trois espaces pour 10000 tribus» (titre du chapitre 7, pp. 107-119). Heureusement, toutesces tribus se partagent entre quelques grands types que je présenterai ici d'abord de façon très schématisće pour essayer ensuite de les situer approximativement sur la carte du touriste. Dans la démarche de l'auteur, le touriste est au centre de l'étude car il porte en lui les codes de perceptions qui l'influencent et le guident dans ses choix et ses comportements touristiques, Il fautdoncs'occuper du touriste d'abord mais il faut aussi s'intéresser à lui pour ses songes culturels, ses valeurs essentielles, son univers symbolique plus que pour ses besoins concrets de biens et services. Les responsables del'offre cesseront d'abord de se préoccuper uniquement de le transporter, le loger et le nourrir pour s'occuper de lui présenter des produits qui parleront à son imaginaire de vacancier nomade.

Le système des trois espaces de la carte du touriste comprenddoncles grands types de tribus touristiques qui se situent ici et là, diversement, sur cette carte.

\section{Les tribus touristiques}

La complexité des genres et des espèces de tribus touristiques est un fait réel qui décourage généralement les tentatives de classement et de définitions. Et cette complexité va croissante à mesure que se poursuit l'apprentissage du nomadisme de vacances. J.-D. Urbain se sert decette notion d'apprentissage pour introduire un premier critère de classification ${ }^{(6)}$. On peut imaginer un continuum qui va d'un tourisme initial à un tourisme expérimental, avec une grande catégorie intermédiaire, celle du tourisme d'initié. Je résume d'abordles traits et les attitudes typiques de ces grandes tribus.

Le tourisme initial: il réfere aux gens qui veulent être touristes comme les autres. On y retrouve des novices qui font des voyages depremière reconnaissance. Pour ceux-lầ, le tourisme est merveilleux et répond à un goût de ressourcement. Leurs voyages sont des pérégrinations pratiquées de fagon rituelle. Ils wont en groupe selon un rite collectif et suivent les circuits comme des cérémonies. Ils sont prudents, peu critiques, recueillis, contemplatifs, comme s'ils pratiquaient un culte, une commémoration. C'est un tourisme de première observation, ni superficiel, ni vulgaire, même si ces touristes sont parfois maladroits. J.-D. Urbain les désignent comme les touristes burons.

Ce huron deviendra plus ou moins vite un discident, à la recherche d'espaces d'innovations. Il cherchera d'abord à déritualiser son voyage par des pratiques typiques: voyager sans règle, sans projet, sans plan, comme un voyage sauvage. Il aura l'air, souvent, du client difficile. Les gens de cette tribu ne veulent plus être touristes; ils sont prêts à entrer dans la seconde grande catégorie.

Le tourisme d'initie.. L'auteur réfere ici à diverses tribus très intéressantes qui ont déjà pris conscience de certains des paradoxes du voyage d'agrément: si on ne peut pas nepas être touriste (puisque tous lesont au moins à leur premier voyage), essayons d'être le moins touriste possible! Ces tribus seront sioukx: gens rusés, insoupçonnables, ils s'appliqueront à vivre incognito, déguisés même, clandestins s'il le faut. Le secret sera leur attitude dominante.

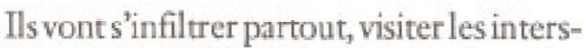
tices et essayer de pratiquer les comporte- ments des explorateurs, des découvreurs. Ils vont réinventer le regard distancié, s'appliquant à redécouvrir l'étrangeté au coeur du quotidien et à apprécier les joies de la marginalité. Cesont des amateurs dehorssaison, allant hors sentiers battus, attirés par les micro-déserts, les enclaves, les lieux obscurs, mystérieux, souterrains, illégaux même.

Les plus initiés des sioux se feront visiteurs des confins. Ces tribus sont comme une case intermédiaire entre les initiés et les expérimentaux. Les confins, ici, sont à entendre symboliquement. On y retrouve d'abord ceux qui veulent fréquenter les confins de la vie sociale, comme le village, espace de convivialité aux antipodes de l'inhumaine ville; on y rencontre aussi les gens qu'attirentlescontinentsoubliés, ceux del'enfance du monde, lieux de naissance (comme l'Irlande), ceux des ancêtres, des vestiges, les pays des dieux (comme la Grèce), ceux des continẹnts rescapés, pays tombaux (comme l'Égypte), lieux de survivance. Enfin, à la limite de cette ca tégorie, l'auteur place les visiteurs des confins des confins, les lieur-limites, lieux de fracture, aux frontièresvilles-campagnes, aux portes des déserts. Là, le vide fascine. Ces lieux-limites sont des attraits irrésistibles pour les touristes; ils annoncent des recyclages nécessaires.

Le tourime expérinental: au pôleopposédu rite se trouve le risque et le goût du péril. Ces tribus - plus récentes souvent - expriment un état actuel de culture. Ses membres sont des téméraires attirés par l'inconnu, l'incroyable, l'impossible.

J.-D. Urbain a identifié, parmi ces tribus, deux sous-groupes psychologiques: un qui veut aller au bout du monde et l'autre, au boutdelui-même. Le premier partira dans l'esprit de Darwin ou des archéologues, prêts à prendre les risques inhérents à ces aventures peu communes; l'autre partira pour tester ses limites, cherchant l'exploit pour l'exploit, prêt à des expériences de survie, à frôler l'épuisement, la mort et ses plaisirs seront proportionnels à l'intensité de ses efforts et aux risques encourus. Ces tribus ont en commun des valeurs d'ascèse, de frugalitế.

Mais ce tourisme expérimental peut être récupérécar, s'il va en enfer (dela jungle, de la ville, etc.), il finitl'aventure dansquelques paradis (les plages des Caraibes après la traversée de la forêt amazonienne). 
TABLEAU 1

\section{TERRITOIRES SYMBOLIQUES}

Tribus

Huron (tourisme initial)

Hurons dissidents

(tourisme déritualisé)

Sioux (tourisme d'initiés)

Visiteurs des confins

Avertis (tourisme aux limites du monde possible) bout de soil
Ascètes (tourisme au
Ville Campagne

- Pour santé, hygiène.

- Un condensé des civilisations.

- Visite la ville visible: monuments, places.

- Villes transparentes à leur valeurs.

- Villes de luxe.

- Pour généalogie, retour aux sources.

- Pays-jardin.

- Calme, paix, sécurité.

Désert

- A la lisière seulement, pour contempler.
- Ville des quartiers,du monde ordinaire.

- Visites sans carte.

- Flănerie
- "Cabotagen agro-touristique de ferme en ferme.

- Asile de la civilisation industrielle.

- Retour aux rythmes naturels.

- Sociabilité hommes-animaux.
- Aller lire les signes du triomphe de la vie.
- Ville la nuit.

- Coins obscurs.

- Agitation des rues.

- Lieux de luxures.

- La ville comme une forêt, une jungle.
- Lieu des maillages secondaires, moins ordonné.

- Réseaux ignorés.

- Les micro-déserts (campagnes abandonnées, etc.).

- Trouverfossiles, traces peu visibles.

- Ruelles.

- Ruines et vestiges.
- Espace d'aventure.

- Recherche de l'humanité primitive.

- Ferme, hameau, village: aux confins de la vie sociale.
- Là où les sociétés s'interrompent.

- Fin des routes.

- Spectacles du bout du monde.

- Survivance.
- Sites de villes disparues.

- Villes impossibles (Brazilia).

- Villes dangereuses (guerre, drogues).
- Archéologie; vestige: présence ancienne.
- Espace du divin.

- Appel de l'étendue.

- Tentation du vide.

- Pure nature: monde inhumain ou pré-humain.
- Ville: lieu d'exploit (N.Y. pour le marathon)
- Périple cyclotouristique (par exemple).
- Exotisme de l'aride.

- Révélation.

- Illumination.

- Mystique du désert.

- Signe de mort.

- Tourisme de purification.

- Arrachementausocial, au culturel. 
Je ne rendrai jamais justice à l'auteur en le résumant ainsi, quant aux tribus touristiques comme quant aux territoires symboliques. Mais nous avons néanmoins une toile de fond pour compléter la présentation du système des espaces.

\section{Les tribus touristiques sur la carte du touriste}

Le tableau 1, ci-dessus, est un essai pour réunir tribus touristiques et territoires symboliques. De façon approximative, le système de J.-D. Urbain est mis en boîte pour les fins de cet article. Une dimension majeure deson analysen'apparaîtpas: celle de l'histoire qui, tout au long de l'ouvrage, nous fait comprendre que ces univers symboliques changent en se transformant, en se complexifiant.

En examinant ce tableau, on trouve une diagonale qui va de la ville, principal territoire du tourisme initial et rituel au désert, lieu privilégié du tourisme expérimental, à risque. Nous nous en inspirerons pour terminer ce long détour à la recherchedela péninsule gaspésienne.

Bas-Saint-Laurent, Gaspésie, llesde-la-Madeleine: quelle carte pour quels touristes?

Ce dernier point n'est qu'un simple essai. Quand je retrouverai tous ceux et celles qui ont participé au colloque de l'ACFAS en mai 1993 ou qui ont contribué au présent numéro de Téoros, je les questionnerai sur toutes mes perceptions nouvelles de leur région, perceptions que cet essai a provoqué. J'en livre maintenant quelquesunes en attendant de connaître les réactions que le cadre d'analyse de J.-D. Urbain fera naître.

Traditionnellement, il me semble que la péninsule gaspésienne fut offerte surtout comme espace de tourisme initial, pour burons et peut-être aussi pour burons dissidents, comme territoire symbolique de la campagne et du désert. Par la suite, je crois qu'on a essayé d'y intéresser les sioux, dans cette campagne et ce désert. Certains visiteurs des confins ont peut-être recherché plus récemmentsa campagne. Aujourd'hui, elle attire occasionnellement, mais de plus en plus semble-t-il, le tourisme expérimental, les avertis plus que les ascètes, amateurs de désert.
Ce diagnosticgrossier conduit quandmême à une condusion: l'espace réel supporte divers territoires symboliques intéressant une variété de tribus touristiques. Peu de celles-ci peuvent trouver, dans cette péninsule, un territoire-ville pour les retenir, mais la symbolique de la campagne est attirante sur toute sa gamme. Il reste sûrement beaucoupà faireencorepour que ce territoire symbolique devienne attirant pour toutes les tribus. Je suis convaincu cependant qu'il est possible d'offrir toute cette gamme dans l'espace géographique qui va des limites de la CUQ au Rocher Percé et aux Iles-de-la-Madeleine.

L'espacesymboliquedudésertserait, quant à lui, l'avenir du tourismedans la péninsule. On comprendra cette hypothèse, évidemment, dans le cadre d'analyse de J.-D. Urbain. La région, dite périphérique, est en fait un territoire symbolique propre aux sioux et aux visiteurs des confins amateurs de désert. Cette région me semble riche aussi de lieux-limites, aux frontières de la campagne et dudésert. Lefleuve, après Québec, appartient à la symbolique de la mer (aspect du désert) et la campagne est toujours coincée, plus ou moins, entre un autre désert: la forêt. Et plus on avance dans la traversée de cette région, plus on va vers un bout du monde, plus leséléments du désert s'affirment et finalement, plus la péninsule peut intéresser le tourime à risques, les avertis comme les ascètes: fin de routes, appel del'étendue, tentation du vide, lieux de survivance, lieux ou des communautés se sont interrompues, archéologie, microdéserts, espaces où l'ascète peut s'arracher du social et du culturel, etc.

Suis-jeen train deréinventer une péninsule gaspésienne? J'ai commencé à relire les guides touristiques de cette région que j'ai visitée quatre ou cinq fois dans ma vie. Je peux dire que je l'ai vu en buron seulement mais j'ai constaté que la promotion de ses territoires symboliques visait de plus en plus d'autres tribus. J'espère que cet essai aidera cette promotion à trouver tous les territoires symboliques que cherchent les sioux, les visiteurs des confins et tous ces nouveaux venus du tourisme expérimental. Au Québec, si le centre est le territoire de la ville, les régionséloignées - etnotamment la péninsule gaspésienne, avec le golfe et ses îles, avec la Côte Nord jusqu'au pays de Vigneault - sont superbement dotées pour séduire toutes les tribus touristiques. $f$

\section{NOTES ET RÉFERENCES}

(1) J.-D. Urbain (1986), Semiotiques compartes of touriste ef du voyageur, in Semiotica, 58, 3/4, pp. 269-286. Mouton de Gruyter, Amsterdam. Voi aussi Semiotics of Touriam, numero spécial de Annals of Tourism Research, vol. 16, no 1. 1969. scus la direction de Dean MacCannell.

(2) Page 116. J-D. Urbain cite F. Beslay a propos du desert: Le désert est le pays de liabsolu et de l'absolu dótachement. II est aussi le pays de l'absoluverité, in La mer oule désert... riend'autre: desert inomades, guemiers, chercheurs d'absolul. paru dans la revue Traverse, p. 47.

(3) Beau concept qu'on n"utilise jamais, applicable non seubment d certaines villes mais aussi d d'autres espaces.

(4) La revue Autrement, consacrée d Montreal, assis du Nord, parue en 1992, fait valoir ce double wisage de bille. A Mbdam (1978: Montral Interdite, Paris, PUF) avait dejâ explore le Montrétal obscur.

(5) Cité par J-D. Urbain, P. 115. L'auteur emprunte a Touwrage de M. Marie et J. Viard, La campagne Inventeb (Arles, Actes Sud, 1989) l'expression: sla campagne est une imention.

(6) A vrai dire, I'auteur n'utilise pas le mot apprentissage et ne cherche pas a faire des classifications au sens formel. Ces grands types supposent qu'on peut apprendre d voyager t travers diverses expériences qui vont d'un tourisme initial at un tourisme expérimental. Mais un méme individu peut vive tous ses voyages awec les attitudes d'un touriste novice, par exemple, alor's qu'un autre franchira des étapes d"experimentation pour s'engager dans des activités plus novatrices. 